\title{
ON CERTAIN CLASSES OF MODULES
}

\author{
K. VARADARAJAN* \\ Dedicated to the memory of Pere Menal
}

\begin{abstract}
Let $X$ be any class of $R$-rnodules containing 0 and closed inder isomorphic images. With any such $\underline{X}$ wc associate thrce classes $\Gamma \underline{X}, F \underline{X}$ and $\Delta \underline{X}$. Thic study of some of the closure properties of these classes allows us to obtain characterization of Artinian modules dualizing results of Chatiers. The theory of Dual Goldic dimension as devclopel by the anthor in some of his earlier work plays a crucial role in the present paper.
\end{abstract}

\section{Introduction}

Thronghont this paper all the rings $R$ we consider will be associative with an identity element, $1_{R} \neq 0$. Lnless otherwise mentioned all the notions such as artinianness, noetherianness will be left sided when we deal with a ring $R$. The modules we consicler will all be unital left modules. In ring theory there are scores of results dealing with the structure of a ring $R$ (resp. of a module $M$ ) assuming certain classes of modules (associated to $M$ ) posses certain properties and vice versa. The results in the present paper are of a similar nature and are an outcome of results proved in $[\mathbf{1}],[2],[3],[4],[5],[6],[8]$ and [9]. In [1] arnong other results $\mathrm{A}$. W. Chatters proves the following:

(i) $R$ is noetherian if and only if every cyclic $R$-module is a direct sum of a projective module and of a noetherian module.

(ii) Given an ordinal $\alpha$, if every cyclic $R$-module is a direct sum of a projective $R$-module and an $R$-rnodule of Krull dimension $\leq \alpha$, then the left $R$-module $R$ has Krull dimension $\leq \alpha+1$.

*While carrying ont, this research the author was visiting the Tata lnsticute of Fundamental Research on invitation from the National Board for IJigher Mathematics of India. Also part of lhis research was carried out at Stanford University where the author spent a portion of his Sabbatical leave. Partial support from NSERC grant A 8225 is gratefully acknowledged. 
In [4] P. F. Smith, Din Van Huynh and Nguyen V. Dung generalize these results of Chatters to module theoretic set up. Let $\underline{X}$ be any class of $R$-modules closed under isomorphic images and satisfying $0 \in \underline{X}$. To any such $\underline{X}$, P. F. Smith et all associate three classes $D \underline{X}, H \underline{X}$ and $E \underline{X}$ and study some of their closure properties under suitable assumptions on $\underline{X}$. 'This not only led them to simpler proofs of the aforementioned results of Chatters, but also to their module theoretic generalizations. Let $\underline{N}, \underline{G}, \underline{K}_{a}$ denote respectively the classes of noctherian modules, finitely generated modules and modules of Krull dimension $\leq \alpha$. The module theoretic generalizations obtained in [4] could be stated as follows.

(iii) $\underline{G} \cap D \underline{N}=\underline{N}$ (generalizing (i)).

(iv) $\underline{G} \cap D \underline{K}_{\alpha} \subseteq \underline{K}_{\alpha+1}$ generalizing (ii)). These are corollaries 3.3 and 2.8 respectively in $[4]$.

Suggested by "duality" in the category $R$-mod of unital left $R$-modules we associate to $\underline{X}$ three more classes $F \underline{X}, \Delta \underline{X}$ and $\Gamma \underline{X}$ (see Section 1 for their definition). The study of some of the closure properties of these classes leads to many interesting results "dualizing" the results of P. F. Smith, Din Van Huynh and Nguyen V. Dung [4]. The object of the present paper is to carry out the study of these closure properties and present proofs of the dual results. For instance one of the results we prove using our methods is the following:

(v) Let $M$ be a semi-perfect module in the sense of [13]. Assume that either $M$ is finitely generated or that $M$ is finitely embedded and $J(M)$ is small in $M$. Then $M$ is artinian if and only if every submodule of $M$ is a direct sum of an injective module and an artinian module.

Actually v) may be regarded as two forms of duals of (iii). $\Lambda$ corollary of $v$ ) is the following characterization of left artinian rings.

(vi) A ring $R$ is left artinian if and only if it is semi-perfect and cvery left ideal of $R$ is a direct sum of an injective left ideal and an artinian left ideal.

\section{The classes $F \underline{X}, \Delta \underline{X}$ and $\Gamma \underline{X}$}

We will be working in the category $R$-rnod of unitary left $R$-modules. The classes $\underline{X}$ of $R$-modules we consider will al ways be assumed to satisfy the following conditions $a$ and $b$.

a. $M \in \underline{X}, M^{\prime} \simeq M \Rightarrow M^{\prime} \in \underline{X}$.

b. $0 \in \underline{X}$. 
To any such $\underline{X}$, P. F. Smith et all [4! associated three classes of modules (though they worked in the category mod- $R$ of right $R$-modules). Before recalling the definition of these classes, we first explain the notation that we will be adopting. For any $M \epsilon R$-mod, we write $N \leq M$ to indicate that $N$ is a subnodule of $M ; N e \frac{5}{e}$ to indicate that $N$ is an essential submodule of $M$ and $N \ll M$ to denote that $N$ is a small submodule of $M$. The three classes $D \underline{X}, H \underline{X}$ and $E \underline{X}$ were defined as follows in [4].

$$
\begin{aligned}
& D \underline{X}=\{M \epsilon R-\bmod \mid N \leq M \Rightarrow M=K \oplus L \text { with } N \leq K \text { and } K / N \epsilon \underline{X}\} . \\
& H \underline{X}=\{M \epsilon R-\bmod \mid N \leq M \Rightarrow M / N \epsilon \underline{X}\} \\
& E \underline{X}=\{M \epsilon R-\bmod \mid N \subseteq M \Rightarrow M / N \epsilon \underline{X}\} .
\end{aligned}
$$

Suggested by "duality" we introduce the following classes:

$$
\begin{aligned}
& \Gamma \underline{X}=\{M \epsilon R-\bmod \mid N \leq M \Rightarrow M=K \oplus L \text { with } K \leq N \text { and } N / K \epsilon \underline{X}\} \\
& F \underline{X}=\{M \epsilon R-\bmod \mid N \leq M \Rightarrow N \epsilon \underline{X}\} \\
& \Delta \underline{X}=\{M \epsilon R-\bmod \mid N \ll M \Rightarrow N \epsilon \underline{X}\} .
\end{aligned}
$$

As in [4] when the ring $R$ is clear from the context, $\underline{M}, \underline{Z}: \underline{P}, \underline{I}, \underline{C}, \underline{G}$, $\underline{N}, \underline{A}, \underline{U}, K_{a}$ will denote respectively the classes of all $R$-modules, the zero modules, projective modules, injective modules, semi-simple modules, finitely gencrated modules, noetherian modules, artinian modules, modules of finite unifom dimension and modules with Krull dimension $\leq \alpha$. Recall [11] that $M \in R$-mod is said to be of dual Goldic dinension $\leq k$ if there exists no surjective map $M \stackrel{\varphi}{\rightarrow} N_{1} \times \ldots \times N_{r}$, with each $N_{i} \neq 0$ and $r \geq(k+1)$. Here $k$ is an integer $\geq 0$. The class of modules of dual Coldic dimension $\leq k$ will be denoted by $\underline{H}_{k}$. We write $\underline{S}$ for the class constituted by the simple modules together with the zero modale. We will mostly be following the notation and teminology in [4]. The class of modules of finite dual Goldie dimension (or corank) will be denoted by $\underline{H}$.

Lemma 1.1. Let $X, \underline{Y}$ be classes of R-modules

(i) If $\underline{X} \subseteq \underline{Y}$ then $L \underline{X \subseteq} \underline{Y}$ where $L$ stands for any one of the symbols $D, H, E, \Gamma, F$ or $\Delta$.

(ii) $F \underline{X}=F(F \underline{X}) \subseteq \underline{X}$.

(iii) $\underline{C} \subseteq \Gamma \underline{X}$.

(iv) $F \underline{X} \subseteq F(\underline{I} \oplus \underline{X}) \subseteq \Gamma(\underline{I} \oplus \underline{X})=\Gamma(\underline{X})=\Gamma(\underline{X}) \subseteq \Delta(\underline{X})$.

(v) $\underline{I} \cap \Gamma \underline{X} \subseteq F(\underline{I} \oplus \underline{X})$.

Proof:

(i) Straight forward. 
(ii) From the very definition of $F \underline{X}$ it is clear that $F \underline{X} \subseteq \underline{X}$. Hence (i) above yields $F(F \underline{X}) \subseteq F \underline{X}$.

Let $M \epsilon F X$ and $N \leq M$. Let $N^{\prime} \leq N$. Then $N^{\prime} \leq M$, hence $N^{\prime} \epsilon \underline{X}$ yielding $N \epsilon F \underline{X}$. This in turn implies that $M \epsilon F(F \underline{X})$; hence $F \underline{X} \subseteq F(F \underline{X})$.

(iii) Let $M \epsilon \underline{C}$ and $N \leq M$. Then $M=N \oplus L$ for some $L \leq M$. Hence the choice $K=N$ fulfills the requirement for $M$ to be in $I^{4} \underline{X}$.

(iv) Since $\underline{X} \subseteq \underline{I} \oplus \underline{X}$, from (i) we get $F \underline{X} \subseteq F(\underline{I} \oplus \underline{X})$.

Let $M \epsilon F(\underline{I} \oplus \underline{X})$ and $N \leq M$. Since $M \epsilon F(\underline{I} \oplus \underline{X})$ we get $N \in \underline{I} \oplus \underline{X}$. Thus $M=0 \oplus M$ and $N / 0 \simeq N \epsilon \underline{I} \oplus \underline{X}$. This means $M \epsilon \Gamma(\underline{I} \oplus \underline{X})$. Hence $F(\underline{I} \oplus \underline{X}) \subseteq \Gamma(\underline{I} \oplus \underline{X})$.

Because of (i), to prove the equality $\Gamma(\underline{I} \oplus \underline{X})=\Gamma \underline{X}$ we have only to show that $\Gamma(\underline{I} \oplus \underline{X}) \subseteq \Gamma \underline{X}$. Let $M \epsilon \Gamma(\underline{I} \oplus \underline{X})$ and $N \leq M$. Then $M=K \oplus L$ with $K \leq N$ and $N / K e \underline{I} \oplus \underline{X}$. From $K \leq N$ we get $N=K \oplus(L \cap N)$; hence $L \cap N \simeq N / K \varepsilon I \oplus X$. This yields $L \cap N=A \oplus B$ with $A \in \underline{I}, B \epsilon \underline{X}$. Since $A \epsilon \underline{I}$ and $A \leq L$ we could write $L=A \oplus C$ with $C \epsilon \underline{M}$. Thus $M=K \oplus L=K \oplus A \oplus C$. Also $K \oplus A \leq N$. Hence $N=K \oplus A \oplus(C \cap N)$. Also $A \leq L \cap N \Rightarrow$ $L \cap N=A \oplus(C \cap N \cap L)=A \oplus(C \cap N)$ since $C \leq L$. From $A \oplus B=L \cap N=A \oplus(C \cap N)$ we get $B \simeq(L \cap N) / A \simeq C \cap N$ yielding $C \cap N \in \underline{X}$. Also $M=K \oplus A \oplus C$ with $K \oplus A \leq N$ and $N /(K \oplus A) \simeq C \cap N \epsilon \underline{X}$. This proves that $M \epsilon \Gamma \underline{X}$. Hence $\Gamma(\underline{I} \oplus \underline{X}) \subseteq \Gamma \underline{X}$.

To complete the proof of iv) we have only to show that $\Gamma X \subseteq$ $\Delta \underline{X}$. Let $M \epsilon \Gamma \underline{X}$ and $N \ll M$. Then $M=K \ominus L$ with $K \leq N$ and $N / K \in X$. From $K \leq N \ll M$ we get $K \ll M$. Since $K$ is a direct summand of $M$ this implies that $K=0$; hence $N \epsilon \underline{X}$ showing that $M \epsilon \Delta \underline{X}$.

(v) Let $M \epsilon \underline{I} \cap \Gamma \underline{X}$ and $N \leq M$. From $M \epsilon \Gamma \underline{X}$ we get $M=K \oplus L$ with $K \leq N$ and $N / K \epsilon \underline{X}$. Then $N=K \oplus(L \cap N)$ yielding $N / K \simeq L \cap N \epsilon \underline{X}$. Also $M \epsilon \underline{I} \Rightarrow K \epsilon \underline{I}$; hence $N \epsilon \underline{I} \oplus \underline{X}$. This means $M \epsilon F(\underline{I} \oplus \underline{X})$ yielding $\underline{I} \cap \Gamma \underline{X} \subseteq F(\underline{I} \oplus \underline{X})$.

Before stating further results let us recall from [4] the definition of $S \underline{X}, Q \underline{X}$ and $P \underline{X}$.

$$
\begin{aligned}
S \underline{X}= & \{N \mid N \leq M, M \in \underline{X}\} . \\
Q \underline{X}= & \{M / N \mid N \leq M, M \in \underline{X}\} . \\
P \underline{X}= & \left\{M \mid \text { there exists a finite chain } 0=N_{0} \leq N_{1} \leq \cdots \leq N_{k}=M\right. \\
& \text { with } \left.N_{i} / N_{i-1} \in \underline{X} \text { for } I \leq i \leq k\right\} .
\end{aligned}
$$

$\underline{X}$ is said to be $S$ (resp $Q$ or $P$ ) closed if $S \underline{X} \subseteq \underline{X}$ (resp. $Q \underline{X} \subseteq \underline{X}$ or $P \underline{X} \subseteq \underline{X})$. 
Lemma 1.2. Let $\underline{X}$ be a class of R-modules. Then

(i) $F \underline{X}, \Delta \underline{X}, \Gamma \underline{X}$ are all $S$-closed.

(ii) If $\underline{X}$ is $S$-closed, then $\underline{X} \subseteq \Gamma \underline{X}$ and $\underline{X C} \subseteq \triangle \underline{X}$.

(iii) $\Gamma \underline{X} \oplus \underline{X}=\Gamma \underline{X}$ if $\underline{X}$ is $\{S, P\}$-closed.

(iv) $F(\underline{I} \oplus \underline{X})=(\underline{I} \oplus \underline{X}) \cap \Gamma \underline{X}$ if $\underline{X}$ is $\{S, P\}$-closed.

(v) $F \underline{X}$ is $Q$-closed if $\underline{X}$ is $Q$-closed.

Proof:

(i) That $F \underline{X}$ is $S$-closed is clear. Let $M \epsilon \Delta \underline{X}$ and $M^{\prime} \leq M$. Let $N^{\prime} \ll M^{\prime}$. Then $N^{\prime} \ll M$ ard hence $N^{\prime} \epsilon \underline{X}$. This means $M^{\prime} \epsilon \Delta \underline{X}$.

Let $M \epsilon \Gamma \underline{X}$ and $M^{\prime} \leq M$. Let $N \leq M^{\prime}$. From $M \epsilon \Gamma \underline{X}$ we get $M=K \oplus L$ with $K \leq N$ and $N / K \in X$. From $K \leq N \leq M^{\prime}$ we get $M^{\prime}=K \oplus\left(M^{\prime} \cap L\right)$. Clearly $N / K \in \underline{X}$; lience $M^{\prime} \in \Gamma \underline{X}$.

(ii) Let $M \epsilon \underline{X}$ and $N \leq M$. Since $\underline{X}$ is $S$-closed we have $N \epsilon \underline{X}$. Thus $M=0 \oplus M$ with $N / 0 \simeq N \epsilon \underline{X}$, yielding $M \epsilon \Gamma \underline{X}$. Hence $\underline{X} \subseteq \Gamma \underline{X}$.

Let $M \epsilon \underline{X} \underline{C}$. Then there exists a $K \leq M$ with $K \epsilon \underline{X}$ and $M / K \epsilon \underline{C}$. Let $N \ll M$. Then $N \leq J(M)$, the Jacobson radical of $M$. If $\eta: M \rightarrow M / K$ denotes the canonical quotient map we get $\eta(N) \leq \eta(J(M)) \leq J(M / K)=0$ since $M / K \epsilon Q$. Hence $N \leq K$. Since $\underline{X}$ is $S$-closed we get $N \epsilon \underline{X}$. Thus $M \epsilon \Delta \underline{X}$ yielding $\underline{X C} \subseteq \Delta \underline{X}$.

(iii) Let $M \epsilon \Gamma \underline{X} \oplus \underline{X}$, say $M=A \oplus B$ with $A \epsilon \Gamma \underline{X}, B \epsilon \underline{X}$. Let $N \leq M$. Since $A \epsilon \Gamma \underline{X}$ we get $A=K \oplus L$ with $K \leq N \cap A$ and $(N \cap A) / K \epsilon \underline{X}$. Thus $M=K \oplus L \oplus B$ and $M / A \simeq B \in \underline{X}$. The exactness of $0 \rightarrow N /(N \cap A) \rightarrow M / A$ together with the $S$-closed nature of $\underline{X}$ yields $N /(N \cap A) \in \underline{X}$. The exactness of $0 \rightarrow(N \cap A) / K \rightarrow$ $N / K \rightarrow N /(N \cap A) \rightarrow 0$ and the $P$-closed nature of $\underline{X}$ imply that $N / K \epsilon \underline{X}$. Hence $M \epsilon \Gamma \underline{X}$, yiclding $\Gamma \underline{X} \oplus \underline{X} \subseteq \Gamma \underline{X}$. The reverse inclusion $\Gamma \underline{X} \subseteq \Gamma \underline{X} \oplus \underline{X}$ is obvious.

(iv) From lemma 1.1(ii) and (iv) we see that $F(\underline{I} \oplus \underline{X}) \subseteq(\underline{I} \oplus \underline{X}) \cap \Gamma \underline{X}$. We can write $M=A \oplus B$ with $A \in I, B \in \underline{X}$. From lemma 1.2(i) we see that $A \in \Gamma \underline{X}$. Let $N \leq M$. Since $A \in \Gamma \underline{X}$ we get $A=K \oplus L$ with $K \leq A \cap N$ and $A \cap N / K \in X$. Hence $M=K \oplus L \oplus B$. From $K \leq N$ we get $N=K \oplus(L \oplus B) \cap N$. Also $A \epsilon I \Rightarrow K \epsilon I$. The exactness of $0 \rightarrow N /(N \cap A) \rightarrow M / A$ and $0 \rightarrow(A \cap N) / K \rightarrow$ $N / K \rightarrow N /(A \cap N) \rightarrow 0$ and $\{S, P\}$-closedness of $X$ immediately yield $N / K \epsilon \underline{X}$. But $N / K \simeq N \cap(L \oplus B)$. Hence $N \epsilon \underline{I} \oplus \underline{X}$, proving that $M c F(\underline{I} \oplus \underline{X})$. Hence $(\underline{I} \oplus \underline{X}) \cap \Gamma \underline{X} \subseteq F(\underline{I} \oplus \underline{X})$.

(v) Let $M \in F \underline{X}$ and $N \leq M$. Any submodule of $M / N$ is of the form $L / N$ with $N \leq L \leq M$. From $M \epsilon F \underline{X}$ we infer $L \epsilon \underline{X}$. Since $\underline{X}$ is $Q$-closed we get $L / N \in \underline{X}$. This implies that $M / N \in F \underline{X}$.

Remarks 1.3. Lemrna 1.1(v) in [4] also asserts that $E \underline{X}$ is $S$-closed 
if $\underline{X}$ is $S$-closed. The dual result if it were true would be that $\Delta \underline{X}$ is $Q$-closed whenever $X$ is $Q$ closed. We now give an easy example to show that the dual result is not true. Let $\underline{Z}$ denote the class consisting of the zero modules in Z-mod. Clearly $\underline{Z}$ is $Q$-closed. Also $\Delta \underline{Z}=\{M \in \mathbf{Z}$-mod $\mid J(M)=0\}$. Clearly $\mathbf{Z} \epsilon \Delta \underline{Z}$, but $\mathbf{Z}_{p^{2}} \notin \Delta \underline{Z}$ for any prime $p$. This shows that $\Delta \underline{Z}$ is not $Q$-closed.

Proposition 1.4. Let $\underline{X}$ be any $\{S, P\}$-closed family of modules. Then I' $X=\Gamma \underline{X} \oplus \underline{X} \oplus(\underline{P} \cap \Gamma \underline{X})$.

Proof: We need only prove the inclusion $\Gamma \underline{X} \oplus \underline{X} \oplus(\underline{P} \cap \Gamma \underline{X}) \subseteq \Gamma \underline{X}$. From lemma 1.2 (iv) we have $\Gamma \underline{X} \oplus \underline{X}=\Gamma \underline{X}$. Hence it suffices to prove that $\Gamma \underline{X} \oplus(\underline{P} \cap \Gamma \underline{X}) \subseteq \Gamma \underline{X}$. Let $M=A \oplus B$ with $A \epsilon \Gamma \underline{X}$ and $B \epsilon \underline{P} \cap \Gamma \underline{X}$. Let $N \leq M$ and $p_{B}: M=A \oplus B \rightarrow B$ the projection onto $B$. From $B \in \Gamma \underline{X}$ we get $B=B_{1} \oplus B_{2}$ with $B_{1} \leq p_{B}(N)$ and $p_{B}(N) / B_{1} \in \underline{X}$. From $B \epsilon \underline{P}$ we get $B_{1} \epsilon \underline{P}$ and $B_{2} \in \underline{P}$. Let $\alpha=p_{B} \mid N \cap\left(A \oplus B_{1}\right): N \cap\left(A \oplus B_{1}\right) \rightarrow$ $B_{1}$. Since $B_{1} \leq p_{B}(N)$ we see that $\alpha: N \cap\left(A \oplus B_{1}\right) \rightarrow B_{1}$ is onto. Since $B_{1} \in \underline{P}$, there exists a splitting $s: B_{1} \rightarrow N \cap\left(A \oplus B_{1}\right)$ of $\alpha$. Let $N^{\prime}=s\left(B_{1}\right)$. Then $N \cap\left(A \oplus B_{1}\right)=N^{\prime} \oplus \operatorname{Ker} \alpha=N^{\prime} \oplus(N \cap A)$. From $A c \Gamma \underline{X}$ we get $A=A_{1} \oplus A_{2}$ with $A_{1} \leq N \cap A$ and $(N \cap A) / A_{1} \in \underline{X}$. Again; $N \cap \vec{A}=A_{1} \oplus\left(N \cap A \cap A_{2}\right)=A_{1} \oplus\left(N \cap A_{2}\right)$ yields $N \cap A_{2} \simeq(N \cap A) / A_{1} \in \underline{X}$. Consider, $p_{B} / A \oplus B_{1}: A \oplus B_{1} \rightarrow B_{1}$. Clearly $s$ is also a splitting for $p_{B} / A \oplus B_{1}$. Since Ker $p_{B} / A \oplus B_{1}=A$ we see that $A \oplus N^{\prime}$ is another internal direct sum representation for $A \oplus B_{1}$. Hence $M=A \oplus B=$ $A \oplus B_{1} \oplus B_{2}=A \oplus N^{\prime} \oplus B_{2}=A_{1} \oplus A_{2} \oplus N^{\prime} \oplus B_{2}$. Since $A_{1} \oplus N^{\prime} \leq N$ we get $N=A_{1} \oplus N^{\prime} \oplus N \cap\left(A_{2} \oplus B_{2}\right)$. Lel $\gamma=p_{B} \mid N \cap\left(A_{2} \oplus B_{2}\right)$ : $N \cap\left(A_{2} \oplus B_{2}\right) \rightarrow B_{2}$. Since $\dot{p}_{B}\left(A_{1} \oplus N^{\prime}\right) \leq B_{1}$ and $B=B_{1} \oplus B_{2}$ we see that $p_{B}(N) \cap B_{2}=p_{B}\left(\left(A_{2} \oplus B_{2}\right) \cap N\right)=$ Image $\gamma$. But $p_{B}(N)=$ $B_{1} \oplus\left(p_{B}(N) \cap B_{2}\right)$; hence image $\gamma=p_{B}(N) \cap B_{2} \simeq p_{B}(N) / B_{1}$ is in $\underline{X}$. Aslo Ker $\gamma=N \cap A_{2} \in \underline{X}$. Since $\underline{X}$ is $P$-closed we get $N \cap\left(A_{2} \oplus B_{2}\right) \epsilon \underline{X}$. Also $N /\left(A_{1} \oplus N^{\prime}\right) \simeq N \cap\left(A_{2} \oplus B_{2}\right) \in \underline{X}$. This shows that $M \in \Gamma \underline{X}$. Thus $\Gamma \underline{X} \oplus \underline{X} \oplus(P \cap \Gamma \underline{X}) \subseteq \Gamma \underline{X}$. This completes the proof of proposition 1.4 .

Lemma 1.5. If $\underline{X}$ is $Q$-closed then $\Delta \underline{X}$ is closed under minimal epimorphic images.

Proof: Let $M \subset \Delta \underline{X}$ and $M \stackrel{\epsilon}{\rightarrow} M^{\prime \prime}$ a minimal epimorphism (i.e. Ker $\epsilon \ll M)$. Then $N^{\prime \prime} \ll M^{\prime \prime} \Leftrightarrow \epsilon^{-1}\left(N^{\prime \prime}\right) \ll M$. In particular $N^{\prime \prime} \ll$ $M^{\prime \prime} \Rightarrow \epsilon^{-1}\left(N^{\prime \prime}\right) \ll M \Rightarrow \epsilon^{-1}\left(N^{\prime \prime}\right) \epsilon \underline{X} \Rightarrow N^{\prime \prime} \epsilon \underline{X}$ (since $\underline{X}$ is $Q$-closed). This proves that $M^{\prime \prime} \in \Delta \underline{X}$.

Before proceeding further we need to recall some definitions and results from $[\mathbf{7}],[\mathbf{1 1}] ;[12],[\mathbf{1 3}]$. Let $N \leq M$. Then $K \leq M$ is called a 
supplement, of $N$ in $M$ if

(a) $K+N=M$ and

(b) $K^{\prime} \leq K, K^{\prime}+N=M \Rightarrow K^{\prime}=K$.

It is known that $K$ is a supplement of $N$ in $M$ if and only if $K+N=M$ and $K \Gamma N \ll K$ (Lemma $6.2 \mathrm{in}[13])$. In [13] we called a module $M$ seniperfect if for every $N \leq M$ there exists a supplenent in $M$ (Definition 6.6 in [13]). In [11] we referred to this as properiy $\left(P_{1}\right)$ for $M$. The module $M$ is said to have property $\left(P_{2}\right)$ if for any $L \leq M, N \leq M$ satisfying $L+N=M$ there exists a supplement $K$ of $N$ in $M$ satisfying $K \leq L$. If $M$ has property $\left(P_{i}\right)$ then any quotient module of $M$ has property $\left(P_{i}\right)$ for $i=1,2$ (Proposition 6.20 in $[13]$ and Proposition 2.29 in $[11\}$ ). Clearly $P_{2} \Rightarrow P_{1}$.

Lemma 1.6. Let $X$ be $Q$-closed and $M \in \Delta X$. Assume further that $M$ has property $\left(P_{1}\right)$. Then every epimorphic image of $M$ is in $\Delta \underline{X}$.

Proof: Let $\eta: M \rightarrow M^{\prime \prime}$ be any epimorphism and $N=$ Ker $\eta$. Let, $K$ be a supplement of $N$ in $M$. Then $K+N=M$ and $K \cap N \ll K$. In particular $\eta / K: K \rightarrow M^{\prime \prime}$ is a minimal epimorphism. From lemma 1.2 (i) we get $K \epsilon \Delta \underline{X}$. Now lemma 1.5 yields $M^{\prime \prime} \epsilon \Delta \underline{X}$.

Example 1.7.

(a) Let $I$ denote the class of torsion abelian groups. In $\mathrm{Z}$-mod, $\underline{T}$ is $\{S, P, Q\}$-closed. In $[4]$ the class $D \underline{T}$ is completely detemined (Proposition 1.6 of [4]). It is easy to see that $E T=M$ and that $H \underline{T}=\underline{T}=F \underline{T}$. For any $M \in \mathbf{Z}$-nod let $J(M)$ denote its Jacobson radical. Since $J(M)$ is the sum of all small submodules of $M$ we see immediately that $\Delta T=\{M \epsilon \mathrm{Z}-\bmod \{,(M) \epsilon T\}$.

From lemma 1.2(i) we know that $\Gamma \underline{T}$ is $S$-closed. Since the only direct summands of $\mathrm{Z}$ are 0 and $\mathrm{Z}$ it follows that $\mathrm{Z} \notin \underline{T}$. Combining this with the $S$-closed nature of $I \underline{T}$ we see that $\Gamma \underline{T} \subseteq \underline{T}$. Also lemma 1.2(ii) implies $\underline{T} \subseteq \Gamma \underline{T}$. Hence $\Gamma \underline{T}=\underline{T}$.

(b) Let $T^{\prime}$ denote the class of torsion free abelian groups. Then $\underline{T}^{\prime}$ is $S$-closed. It is trivial to see that $F \underline{T}^{\prime}=\underline{T}^{\prime}$.

Suppose $M e \Gamma \underline{T}^{\prime}$. Since the only torsionfree factor gromp of a torsion abelian group is 0 we see that any $N \leq t(M)$ is a direct summand of $M$ (here $t(M)$ denotes the torsion subgroup of $M$ ). It follows that any $N \leq t(M)$ is a direct summand of $t(M)$ and that $t(M)$ itself is a direct summand of $M$. Thus $t(M) \subset C$ and $M=t(M) \oplus L$ with $L \in \underline{I^{\prime}}$. 'This yields $I^{\prime} \subseteq C \oplus T^{\prime}$. Also $A \in C \Leftrightarrow A=t(A)$ and $t_{p}(A)$ is a vector space over $\mathrm{Z}_{p}$ for every prime $p$. Let $M=A \oplus B$ with $A \in \underline{C}$ and $B \in \underline{T}^{\prime}$. Let $N \leq M$. Then $t(N) \leq t(M)=A$. Since $A \epsilon \underline{C}$ we get $A=t(N) \oplus L$ and 
both $t(N)$ and $L$ will be in $\underline{C}$. From $M=A \oplus B=t(N) \oplus L \oplus B$ and $N / t(N) \epsilon \underline{T}^{\prime}$ we see that $M \epsilon \Gamma \underline{T}^{\prime}$. Hence $C \odot \underline{T}^{\prime} \subseteq \Gamma \underline{T}^{\prime}$. Using the reverse inclusion already proved we get

$$
\Gamma \underline{T}^{\prime}=\underline{C} \oplus \underline{T}^{\prime} .
$$

From lemma 1.1 (iv) we have $\Gamma \underline{T}^{\prime} \subseteq \Delta \underline{T}^{\prime}$. We will actually give a conpletc characterization of the class $\Delta T^{\prime}$ from which it will follow immediately that the inclusion $\Gamma T^{\prime} \subseteq \Delta T^{\prime}$ is a strict inclusion.

Let $M \epsilon \Delta \underline{T}^{\prime}$. Suppose for some prime $p$, the $p$-primary torsion $t_{p}(M)$ of $M$ is non-zero. Then there exists a copy of $Z_{p}$ in $t_{p}(M)$. Suppose $N \leq M$ satisfies $\mathbf{Z}_{p}+N=M$. Either $N \cap \mathbf{Z}_{p}=\mathbf{Z}_{p}$ or $N \cap \mathbf{Z}_{p}=0$, in the former case $N=M$ and in the latter case $M=N \oplus \mathbf{Z}_{p}$. If for all $N \leq M$ satisfying $\mathrm{Z}_{p}+N=M$ we have $N=M$, then $\mathrm{Z}_{p} \ll M$ and this contradicts the assumption that $M \epsilon \Delta T^{\prime}$. Hence $M=Z_{p} \oplus N$ for some $N \leq M$. Thus we have shown that if $t_{p}(M) \neq 0$, any copy of $Z_{p}$ in $t_{p}(M)$ is a direct summand of $M$. In particular this implies that there are no elements of order $p^{2}$ in $t_{p}(M)$, hence $t_{p}(M)$ is a vector space over $\mathrm{Z}_{p}$. Hence $t(M)=\oplus_{p} t_{p}(M)$ is in $\underline{C}$.

We claim that

$$
\begin{aligned}
& \Delta \underline{T}^{\prime}=\left\{M \epsilon \mathrm{Z}-\bmod / \operatorname{ary} \mathrm{Z}_{p} \leq M\right. \\
& \text { for any prime } p \text { is a direct summand of } M\} .
\end{aligned}
$$

Because of the observations in the earlier paragraph, to prove (4) we have only to show that if $M \in \mathrm{Z}$-mod has the property mentioned in the right hand side of (4) and if $N \ll M$ then $N \in \underline{T}^{\prime}$. If on the contrary there is an $N \ll M$ with $N \notin \underline{T}^{\prime}$, then $t_{p}(N) \neq 0$ for some prime $p$. Then there is a copy of $Z_{p}$ in $t_{p}(N)$. Since $N \ll M$ it will follow that this copy of $\mathrm{Z}_{p}$ is small in $M$. However, any $\mathrm{Z}_{p} \leq M$ being a direct summand of $M$ cannot be small in $M$.

From (4) we see that

$$
M=\prod_{p} \mathrm{Z}_{p}
$$

(direct product over all primes) is in $\Delta \underline{T}^{\prime}$. However, $t(M)=\oplus_{p} \mathrm{Z}_{p}$ and it is well-known that $t(M)$ does not split off from $M$. Hence $M \notin \Gamma \underline{T}^{\prime}$. This proves that the inclusion $\Gamma^{\prime} \underline{T}^{\prime} \subseteq \Delta \underline{T}^{\prime}$ is strict.

\section{Study of $\Delta \underline{X}$ when $\underline{X}=\underline{A} \cap \underline{H}_{k}$}

For results on dual Goldie dimension or corank the reader may refer to [7], [11]. As already remarked in [11]: if the dual Goldie dimension 
of $M$ is infinite we cannot assert that tlere exists a surjective map $\varphi$ : $M \rightarrow \Pi_{i=1}^{\infty} N_{i}$ with each $N_{i} \neq 0$. (See Proposition 1.6 in [11]). All we can assert in this case is that, given any integer $d \geq 1$ we can find a certain surjection $\theta: M \rightarrow \Pi_{j=1}^{d} L_{j}$ with each $L_{j} \neq 0$ (the modules $L_{j}$ in general will depend on $d$ ). This different behaviour of dual Goldie dimension as compared to Goldie dimension necessitates many changes in the formulation and in the proofs of $r$ esults dual to those obtained in Section 2 of [4] where the theory of Goldie dimension plays a crucial role. We first observe that the class $\underline{H}_{k}$ is $Q$-closed.

Lemma 2.1. Let $\underline{X}$ be $Q$-closed with $\underline{X} \subseteq \underline{H}_{k}$. Let $M \epsilon \Delta \underline{X}$ and $N \leq M$ satisfy $N+\bar{J}(M)=M$. Assume that $M$ has property $\left(P_{1}\right)$. Then $M / N \in \underline{H}_{k}$.

Proof: Let $\eta: M \rightarrow M / N$ denote the quotient map. From $N+$ $J(M)=M$ we get $\eta(J(M))=M / N$. Hence $J(M / N)=M / N$. Suppose if possible that $M / N$ has dual Goldie dimerision $>k$. Then there cxists a surjection $\varphi: M / N \rightarrow A_{1} \times \cdots \times A_{\ell}$ with $\ell>k$ and each $A_{j} \neq 0$. From $J(M / N)=M / N$ we get $J\left(A_{j}\right)=A_{j}$ for $1 \leq j \leq \ell$. Since $J\left(A_{j}\right)=A_{j} \neq 0$ and $J\left(A_{j}\right)$ is the sum of all small subrnodules of $A_{j}$ we see that there exists a $B_{j} \ll A_{j}$ wilh $B_{j} \neq 0$. Then $B_{1} \times \cdots \times B_{\ell} \ll$ $A_{1} \times \cdots \times A_{\ell}$. From lemma 1.6 we get $A_{1} \times \cdots \times A_{\ell} \epsilon \underline{\Delta}$. This implies $B_{1} \times \cdots \times B_{\ell} \in \underline{X}$. This contradicts the assumption that $\underline{X} \subseteq \underline{H}_{k}$, since corank $B_{1} \times \cdots \times B_{\ell} \geq \ell>k$.

Corollary 2.2. Suppose $\underline{X}$ is Q-closed and $\underline{X} \subseteq \underline{H}_{k}$. Let $M \epsilon \Delta \underline{X}$. Suppose $M$ has property $\left(P_{1}\right)$ and satisfies $J(M)=M$. Then $M \in \underline{H}_{k}$.

Proof: Choose $N=0$ in lemma 2.1 .

Proposition 2.3. Let $\underline{X}$ be $Q$-closed with $\underline{X} \subseteq \underline{H}_{k}$. Let $M \epsilon \Delta \underline{X}$ and assume that $M$ has property $\left(P_{1}\right)$. Then there exists an $N \epsilon \underline{X}$ such that $M / N=B \oplus H$ with $B \epsilon \underline{C}$ and $H \epsilon \underline{H}_{k} \cap \Delta \underline{X}$.

Proof: Let $L$ be a supplement of $J(M)$ in $M$. Then $L+J(M)=M$ and $L \cap J(M) \ll L$. Also $L /(L \cap J(M)) \simeq M / J(M) \in \Delta \underline{X}$ by lemma 1.6 . Since $\Delta \underline{X}$ is $S$-closed (lemma $1.2(\mathrm{i})$ ) we get $L \epsilon \Delta \underline{X}$. From $L \cap J(M) \ll L$ we get $L \cap J(M) \in \underline{X}$. Since $M / J(M)$ has property $\left(P_{1}\right)$ (Proposition 6.1 in [13]) and $J(M / J(M))=0$ from proposition 3.3 in [11] we see that $M / J(M) \in \underline{C}$. Hence $L /(L \cap J(M)) \in \underline{C}$. From lemma 2.1 we get $M / L \in \underline{H}_{k}$. If we set $N=L \cap J(M)$ we get $N \varepsilon \underline{X}$ and $0 \rightarrow L / N \rightarrow M / N \rightarrow M / L \rightarrow 0$ exact. 
Since $L+J(M)=M$ any $x \epsilon M$ can be written as $\ell_{x}+u_{x}$ with $\ell_{x} \epsilon L$ and $u_{x} \epsilon J(M)$. If $x=\ell_{x}+u_{x}=\ell_{x}^{\prime}+u_{x}^{\prime}$ are two such expressions, we have $\ell_{x}-\ell_{x}^{\prime}=u_{x}^{\prime}-u_{x}$ is in $L \cap J(M)=N$. Thus the element $\bar{\ell}_{x}$ in $L / N$ represented by $\ell_{x}$ depends only on $x$. Moreover, if $x \in N, x=0+x$ is such an expression, hence $\bar{\ell}_{x}=0$. It follows that one gets a welldefined map $\alpha: M / N \rightarrow L / N$ given by $\alpha(x+N)=\ell_{x}+N$. It is easily checked that $\alpha$ yields a splitting of the inclusion $L / N \hookrightarrow M / N$.

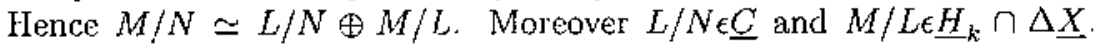
That $M / L \epsilon \Delta \underline{X}$ is a consequence of lemma 1.6. Set $B=L / N$ and $H=M / L$.

Proposition 2.4. Let $\underline{X}$ be a $\{P, Q, S\}$-closed class of $R$-modules. Let $M \epsilon R$ - $\bmod$ and $N \leq M$ satisfy $N c \underline{X}$ and $M / N=B \oplus\left(H_{1}+\cdots+H_{k}\right)$ with $B \in \underline{C}$ and $H_{1}+\cdots+H_{k}$ an irredundant sum of hollow modules (this sum need not be direct). Suppose $H_{i} \epsilon \Delta \underline{X}$ for $1 \leq i \leq k$. Then $M \epsilon \Delta \underline{X}$.

Proof: Let $K \ll M$. We have to show that $K \epsilon \underline{X}$. Let $\eta: M \rightarrow M / N$ denote the quotient map. Writing $H$ for $H_{1}+\cdots+H_{k}$ we have $M / N=$ $B \oplus H$. Since $K \ll M$ we get $K \leq J(M)$, hence $\eta(K) \leq \eta(J(M)) \leq$ $J(M / N)=J(H)$ since $B \epsilon \underline{C}$.

We claim that $\eta(K) \cap H_{i} \neq H_{i}$ for each $i$ in $1 \leq i \leq k$. In fact from $H_{1}+\cdots+\hat{H}_{i}+\cdots+H_{k} \neq H$ we get $L=\eta^{-1}\left(B \oplus\left(H_{1}+\cdots+\hat{H}_{i}+\cdots+\right.\right.$ $\left.\left.H_{k}\right)\right) \neq M$. Here $H_{1}+\cdots+\hat{H}_{i}+\cdots+H_{k}$ denotes the sum of the $H_{j}^{\prime} \mathrm{s}$ with $j \neq i$. Jf $\eta(K) \cap H_{i}=H_{i}$ we would have $K+L=M$ contradicting the fact that $K \ll M$. Thus $\eta(K) \cap H_{i} \neq H_{i}$. Since $H_{i}$ is hollow we get $\eta(K) \cap H_{i} \ll H_{i}$. Since $H_{i} \epsilon \Delta \underline{X}$, this yields $\eta(K) \cap H_{i} \epsilon \underline{X}$.

For each $i$ in $1 \leq i \leq k$ let $A_{i}=\eta(K) \cap\left(H_{1}+\cdots+H_{i}\right)$. By induction on $i$ we will show that $A_{i} \epsilon X$ for $1 \leq i \leq k$. We have seen already that $A_{1}=\eta(K) \cap H_{1}$ is in $\underline{X}$. Let $i \leq k-1$ and assume that $A_{i} \epsilon \underline{X}$. From $A_{i+1} / A_{i} \simeq \frac{\eta(K) \cap H_{i+1}}{A_{i} \cap H_{i+1}}$, since $\underline{X}$ is $Q$-closed we get $A_{i+1} / A_{i} \in \underline{X}$. Since $\underline{X}$ is $P$-closed and $A_{i}$ is already in $X$ we get $A_{i+1} \in X$, thus completing the inductive step. Hence $A_{k}=\eta(K) \in \underline{X}$. Also $N \epsilon \underline{X}$ implies $N \cap K \in \underline{X}$ since $\underline{X}$ is $S$-closed. The exactness of $0 \rightarrow N \cap K \rightarrow K \rightarrow \eta(K) \rightarrow 0$ together with the $P$-closedness of $\underline{X}$ yields $K \epsilon \underline{X}$.

Remarks 2.5.

(i) It is clear that any non-artinian module $M$ will contain a proper non-artinian submodule. Hence il $M$ is a module with the property that $N \epsilon \underline{A}$ for all $N \subsetneq M$ then $M$ itself is in $\underline{A}$. In particular a hollow module $H$ will satisfy $H \subset \Delta \underline{A}$ if and only if $H \in \underline{A}$.

(ii) The classes $\underline{A}$ and $\underline{N}$ are $\{P, Q, S\}$-closed. Hence proposition 2.4 is valid when $\underline{X}=\underline{A}$ or $\underline{N}$. 
(iii) Any $M \in A$ or any $M \in C$ or any hollow module $M$ has property $\left(P_{2}\right)$. Modules with finite spanning dimension in the sense of $P$. Fleury (Section 4 of [11]) have property $\left(P_{2}\right)$. All artinian modules have finite spanning dimension and hence finte corank.

The following results proved in [7], [11] will be needed later in our present paper

(iv) If $M \in \underline{H}_{k}$ has property $\left(P_{2}\right)$ then $M$ can be written as an irredundant sum $H_{1}+\cdots+H_{r}$ of hollow modules with $r \leq k$. This is Theotem 2.39(1) in [11].

(v) If $M=H_{1}+\cdots+H_{r}$ wilh $H_{i}$ hollow, then corank $M \leq r$. This is Proposition 1.7 in [7]. For this part, we need not assume that $M$ has property $\left(P_{2}\right)$.

Let us denote the class of nodules with property $\left(P_{i}\right)$ by $M_{i}(i=1,2)$. We can state one of our main results as follows.

Theorem 2.6. We have following inclusions.

(a) $(\underline{C} \oplus A) A \subseteq \Delta \underline{A}$.

(b) $\underline{M}_{2} \cap \Delta\left(\underline{A} \cap \underline{H}_{k}\right) \subseteq\left(\underline{C} \oplus \underline{A} \cap \underline{H}_{k}\right) \underline{A} \cap \underline{H}_{k}$.

Proof: (a) Let $M \epsilon(\underline{C} \oplus \underline{A}) \underline{A}$. Then there exists an $N \leq M$ with $N \in \underline{A}$ and $M / N=B \oplus L$ wilh $B \in \underline{C}$ and $L \in \underline{A}$. Since $L$ has $\left(P_{2}\right)$ and of finite dual Goldie dimension we can write $L=H_{1}+\cdots+H_{r}$ arl irredundant: sum of hollow modules (see iv) in remark 2.5). From $L \in \underline{A}$ we see that $H_{i} \in \underline{A}$. Since $\underline{A}$ is $S$-closed, we have $\underline{A} \subseteq \Delta \underline{A}$. From proposition 2.4 we see that $M \in \Delta \underline{A}$.

(b) The class $\underline{A} \cap \underline{H}_{k}$ is $Q$-closed. Let $M \epsilon \underline{M}_{2} \cap \Delta\left(\underline{A} \cap \underline{H}_{k}\right)$. From proposition 2.3, there exists an $N \in \underline{A} \cap \underline{H}_{k}$ such that $M / N=B \oplus H$ with $B \epsilon \underline{C}$ and $H \epsilon \underline{H}_{k} \cap \Delta\left(\underline{A} \cap \underline{H}_{k}\right)$. Since $M$ has $\left(P_{2}\right)$ it follows from proposition 2.29 in [11 that $H$ has $\left(P_{2}\right)$. Hence $H=H_{1}+\cdots+H_{r}$ an irredundant sum of hollow modules with $r \leq k$. From lemma 1.2(i) each $H_{j}$ is in $\Delta\left(\underline{A} \cap \underline{H}_{k}\right)$. In particular $H_{j} \in \Delta(\underline{A})$. From remark 2.5 (i) we see that $H_{j} \in \underline{A}$. Thus $H \epsilon \underline{A} \cap \underline{H}_{k}$ by remark 2.5). This proves (b).

Stated in words Theoren 2.6 (b) takes the following form.

Theorem 2.7. Let $M$ be a module with property $\left(P_{2}\right)$. Suppose every small sibrhodule of $M$ is artinian and of datal Goldie dimension $\leq k$. Then there exists an artinian submodule $N$ of $M$ with conank $N \leq k$ such that $M / N=B \otimes L$ with $B$ semi-simple and $L$ artinitan of conank $\leq k$.

Corollary 2.8. Suppose $M$ is a module with property $\left(P_{2}\right)$ and of 
finite corank. Sizppose every small submodule of $M$ is artinion and of dual Goldie dimension $\leq k$ for some fixed integer $k$. Then $M$ is artinian.

Proof: From the above theorem, there exists an artinian submodule $N$ of $M$ such that $M / N=B \oplus L$ with $B \epsilon \underline{C}$ and $L \epsilon \underline{A}$. Now, corank $B \leq$ corank $M / N \leq$ corank $M<\infty$. A semi-simple module has finite corank if and only if it is semi-simple artinian. It follows that $B \in \underline{A}$ and hence $M \epsilon \underline{A}$.

We have a variant of corollary 2.8 which is actually easier to prove.

Proposition 2.9. Let $M$ be a finitely generated module with property $\left(P_{1}\right)$. Then $M \epsilon \Delta \underline{A}$ if and only if $M$ is artinian.

Proof: Since $M / J(M)$ has property $\left(P_{1}\right)$ and $J(M / J(M))=0$ it follows that $M / J(M) \in \underline{C}$. Since $M$ is finitely generated it follows that $M / J(M)$ is semi-simple artinian. Since $M$ is finitely generated we also have $J(M) \ll M$. Thus $M \epsilon \Delta \underline{A} \Rightarrow J(M) \in \underline{A}$. From $M / J(M) \in \underline{A}$ we get $M \in \underline{A}$. Conversely, we have already observed that $\underline{A} \subseteq \Delta \underline{A}$.

Proposition 2.10. Suppose $\underline{X}$ is a $\{P, Q\}$-closed class satisfying $\underline{S} \subseteq$ $\underline{X} \subseteq \underline{H}$. Suppose $M$ is a finitely enbedded module with property $\left(P_{1}\right)$ satisfying $M \in \Gamma \underline{X}$ and $J(M) \in \underline{X}$. Then $M \in \underline{X}$.

Proof: We will abbreviate finitely generated as f.g and finitely embedded as f.e. We have $M / J(M) \in \underline{C}$ because $M / J(M)$ has $\left(P_{1}\right)$ and $J(M / J(M))=0$. If we show that $M / J(M)$ is f.g it will follow from $\underline{S} \subseteq \underline{X}$ and the $P$-closed nature of $\underline{X}$ that $M / J(M) \epsilon \underline{X}$. Again $J(M) \in \underline{X}$ and $M / J(M) \epsilon \underline{X}$ will yield $M \epsilon \underline{X}$.

Suppose on the contrary $M / J(M)$ is not f.g. Then $M / J(M)=V_{1} \oplus V_{2}$ with $V_{1}, V_{2}$ semi-simple and each not f.g. Since a non f.g serni-simple module does not have finite corank we see that $V_{i} \notin \underline{X}$ for $i=1,2$. Let $\eta: M \rightarrow M / J(M)$ denote the quotient map and $L_{1}=\eta^{-1}\left(V_{1}\right)$. Since $V_{1} \notin \underline{X}$ and $\underline{X}$ is $Q$-closed it follows that $L_{1} \notin \underline{X}$. From $M \epsilon \Gamma \underline{X}$ we get $M=N_{1} \oplus W_{1}$ with $N_{1} \leq L_{1}$ and $L_{1} / N_{1} \epsilon \underline{X}$. From $L_{1} \notin \underline{X}$ we get, $N_{1} \neq 0$. From $J(M)=J\left(N_{1}\right) \oplus J\left(W_{1}\right)$ we get $J(M) \cap N_{1}=$ $J\left(N_{1}\right)$ and $J(M) \cap W_{1}=J\left(W_{1}\right)$. This yields $M / J(M)=\left(N_{1} / J\left(N_{1}\right)\right) \oplus$ $\left(W_{1} / J\left(W_{1}\right)\right)$. Also $N_{1} / J\left(N_{1}\right)=N_{1} / J(M) \cap N_{1} \leq L_{1} / J(M)=V_{\mathrm{l}}$. Since $M / J(M)=V_{1} \oplus V_{2}$ and $V_{2}$ is not f.g and $N_{1} / J\left(N_{1}\right) \leq V_{1}$ it follows that $W_{1} / J\left(W_{1}\right)$ is not I.g. Since $W_{1}$ is a direct summand of $M$ we see that $W_{1}$ is f.e. Since $W_{1}$ is a quotient of $M$ we see that $W_{1}$ has property $\left(P_{1}\right)$. From lemma $1.2(\mathrm{i})$, since $W_{1} \leq M$ we get $W_{1} \in \Gamma \underline{X}$. Since $\underline{X}$ is $Q$-closed, from $J(M) \in \underline{X}$ we get $J\left(W_{1}\right) \in \underline{X}$. Thus $W_{1}$ satisfies all the 
conditions imposed on $M$ and further $W_{1} / J\left(W_{1}\right)$ is not l.g. Hence the same arguments as above will yield a decomposition $W_{1}=N_{2} \oplus W_{2}$ with $N_{2} \neq 0, W_{2}$ f.e with properiy $\left(P_{1}\right), W_{2} \epsilon \Gamma \underline{X}, J\left(W_{2}\right) \in \underline{X}$ and $W_{2} / J\left(W_{2}\right)$ semi-simple but not $f . g$. Iteration of this argument yields for any integer $k \geq 1$ a direct sum decomposition $M=N_{1} \oplus \cdots \oplus N_{k} \oplus W_{k}$ with each $N_{j} \neq 0$. This means that the Goldie dimension of $M \geq k$ for every integer $k \geq 1$. However, any f.e module trivially has finite Goldie dimension. This contradiction shown that $M / J(M)$ las to be f.g thus cornpleting the proof of proposition 2.10 .

\section{Dual of Chatters' result}

As stated in the introduction Chatters has proved that if every cyclic $R$-module is a direct sum of a projective module and a noetherian module, then $R$ is noetherian. The module theoretic generalization obtained in [4] asserted that $\underline{G \cap D N}=\underline{N}$. In this section we will prove two forms of duals for the above mentioned result.

Theorem 3.1. Let $M \epsilon R$-mod satisfy the condition that every submodule of $M$ is the direct sum of an injective module and an artinian module. Suppose further that $M$ satisfies one of the following conditions:

(i) $M$ has $\left(P_{1}\right)$ and is $f . g$ or

(ii) $M$ has $\left(P_{1}\right)$, is f.e and $J(M) \ll M$.

Then $M \epsilon \underline{A}$.

Proof: Part of our hypothesis could be rephrased as $M \in F(I \oplus \underline{A})$. Since $\underline{A}$ is $\{S, P\}$-closcd, from lemma 1.2 (iv) we infer that $M \in(\underline{I} \oplus \underline{A}) \cap \Gamma \underline{A}$. Lemma 1.1(iv) yields $\Gamma \underline{A} \subseteq \Delta \underline{A}$. It follows that $M \in \Delta \underline{A}$.

In case (i) is valid, proposition 2.9 immediatcly yields $M \in A$. In case (ii) is valid, the assumption that $J(M) \ll M$ implies that $J(M) \epsilon \underline{A}$. Then proposition 2.10 yields $M \in \underline{A}$.

Converscly, if $M \in \underline{A}$ every $N \leq M$ satisfies $N \in \underline{A}$. Thus $N=0 \oplus N$ is an expression for $N$ as the direct sum of an injective module and an artinian module.

Corollary 3.2. Let $R$ be a semi-perfect ring. Then every left ideal of $R$ is a direct sum of an injective left ideal and an artinian left ideal if and only if $R$ is left artinion.

Proof: This is an immediate consequence of theorem 3.1(i). 


\section{Remarks 3.3.}

(a) We have already observed that if $M \epsilon R$-mod satisfies the condition that $N \in \underline{A}$ for all $N \subsetneq M$, then $M \in \underline{A}$. The module $\mathbf{Z}_{p_{\infty}}$ in $\mathbf{Z}$-mod has the property that every $N \subsetneq \mathrm{Z}_{p_{\infty}}$ satisfes $N C \underline{N}$ but $\mathrm{Z}_{p_{\infty}}$ itself is not in $\underline{N}$.

(b) Dually if $M \epsilon R$-mod satisfies the condition that $M / N \epsilon N$ for all $0 \neq N \subseteq M$ then $M \epsilon \underline{N}$. Z $\mathbf{Z}$ in $\mathbf{Z}$-mod satisfies the condition that for any $0 \neq N \subseteq \mathbf{Z}$, the factor module $\mathbf{Z} / N$ is artinian but $\mathbf{Z}$ itself is not artinian.

(c) Recall that a module $M$ is said to be Hopfian (resp. co-Hopfian) if every surjective (resp. injective) map $f: M \rightarrow M$ is an isomorphism. It is well-known that any $M \in \underline{N}$ is Hopfian (resp, any $M \in \underline{A}$ is co-Hopfian). Presently we will see that Hopfian (resp. co-Hopfian) modules satisfy the property stated in (b) (resp. a)).

Proposition 3.4. Suppose $M \in R$-mod satisfies the condition that $M / N$ is Hopfian for every $0 \neq N \subseteq M$. Then $M$ itself is Hopfian.

Proof: Suppose on the contrary $M$ is not Hopfian. Then there exists a surjection $f: M \rightarrow M$ which is not an isomorphism. Let $N=$ ker $f$. Then $0 \neq N$ and $f$ induces an isornorphism $\bar{f}: M / N \rightarrow M$. If $\eta: M \rightarrow M / N$ denotes the canonical quotient map, then $M / N \stackrel{\text { nof }}{\longrightarrow} M / N$ is a surjection which is not an isomorphism, contradicting the Hopfian nature of $M / N$.

Proposition 3.5. Suppose $M \epsilon R$-mod satisfies the condition that $N$ is co-Hopfian for any $N \subsetneq M$. Then $M$ itself is co-Hopfian.

Proof: Suppose on the contrary $M$ is not co-Hopfian. Then there exists an injective map $g: M \rightarrow M$ which is not an isomorphism. Let $N=$ Image $g$. Then $N \subseteq M$ and $g$ induces an isomorphism $\bar{g}: M \rightarrow N$. Then $\bar{g} / N: N \rightarrow N$ is an injective map which is not an isomorphism contradicting the co-Hopfian nature of $N$.

It is casy to see that if $M / N$ is f.g for every $0 \neq N \subseteq M$ then $M$ itself is f.g. We have the following dual result.

Proposition 3.6. Let $M \in R$-mod satisfy the condition that for any $N \subsetneq M, N$ is f.e. Then $M$ itself is f.e.

Proof: We may assume $M \neq 0$. We first show that Soc $M$ is f.g. It this is not the case we will have Soc $M=\oplus_{\alpha, x,} S_{\alpha, x}$ with each $S_{\alpha}$ simple and $J$ infinite. Let $J^{\prime}=J-\left\{\alpha_{0}\right\}$ where $\alpha_{0}$ is a chosen element in $J$. Then 
$N=\oplus_{c r e} S_{C .} \subsetneq$ Soc $M \subseteq M$. Hence by assumption $N$ is f.e. This means Soc $N$ has to be a direct sum of finitely many simple modules. But Soc $N=\oplus_{\alpha x J^{\prime}} S_{\alpha}$ with $J^{\prime}$ infinile; a contradiction. This contradiction shows that $\operatorname{Soc} M=\Theta_{\alpha c} S_{\alpha}$ with each $S_{\alpha}$ simple and $J$ finite.

Next we claim that Soc $M \neq 0$. Either $M$ is simple in which case $0 \neq M=\operatorname{Soc} M$ or there exists an element $x \neq 0$ in $M$ with $N=R x \subseteq$ $M$. Then $0 \neq N$ and $N$ is fe by assumption. Hence $E(N)=E(\operatorname{Soc} N)$ yielding $\operatorname{Soc} N \neq 0$. From $\operatorname{Soc} N \leq \operatorname{Soc} M$ we see that $\operatorname{Soc} M \neq 0$. Now we will show that $E(M)=E(\operatorname{Soc} M)$. This will prove that $M$ is f.e. If $E(M) \neq E(\operatorname{Soc} M)$ we can write $E(M)=E \oplus E(\operatorname{Soc} M)$ with $0 \neq E \epsilon I$. Let $0 \neq x \in E$. Then $N=R x \cap M \neq 0$ since $M \grave{e} E(M)$. Also $x \in E \Rightarrow R x \cap$ Soc $M=0$. If $N=M$, we would have $R x=M$, hence $\operatorname{Ran}$ Soc $M=$ Soc $M \neq 0$. This shows that $N \neq M$. Hence $N$ is f.e. Since $N \neq 0$, we sec that $\operatorname{Soc} N \neq 0$. Then $\operatorname{Soc} N \leq \operatorname{Soc} M$ will yield $N \cap \operatorname{Soc} M \geq$ Soc $N \neq 0$. This contradicts $\operatorname{Ren} \operatorname{Soc} M=0$. This contradiction shows that $E(M)=E(\operatorname{Soc} M)$.

A ring $R$ is said to be directly finite if $x \in R, y \in R, x y=1 \Rightarrow y x=1$. It is well-known and easy to sce that $R$ is Hopfan in $R$-rnod if and only if $R$ is directily finite [14]. It is shown in our carlier paper that $R$ is co-Hiopfian in $R$-mod if and only if every left regular element $a$ of $R$ is a two sided unit (Proposition 1.4 in [14]). We are led to the following questions from the results in our present paper.

(1) If every cyclic $R$-module is a direct sum of a projective module and a Hopfian module is it true that $R$ is directly finite? More generally what can we say about a module $M$ which satisfies the condition that every quotient of $M$ is a direct sum of a projective module and a Hophan module?

(2) If every left ideal of $R$ is a direct sum of an injective left ideal and a co-Hopfian left ideal is $R$ co-Hopfian in $R$-mod? More generally if $M \in R$-mod satisfies the condition that every submodule of $M$ is a clirect sum of an injective module and a co-Hopfian module what can we say about the structure of $M$ ? Also the study of the following classes may prove to be fruitul.

$$
\begin{aligned}
L \underline{X} & =\{M \epsilon R-\bmod \mid N \underline{\leq} M \Rightarrow N \epsilon \underline{X}\} \\
V \underline{X} & =\{M \epsilon R-\bmod \mid N \ll M \Rightarrow M / N \epsilon \underline{X}\} .
\end{aligned}
$$

Concerming these classes the following are eusy to prove. $L \underline{X} \subseteq \underline{X}$ and $V \underline{X} \subseteq \underline{X}$. In lact $L \underline{X} \subseteq \underline{X}$ is immediate from the fact that $M \stackrel{\leq}{e} M$ and $V \underline{X} \subseteq \underline{X}$ is immediate from the fact that $0 \ll M$. If $\underline{T}$ denotes the class of torsion abelian groups we get $L \underline{T}=V \underline{T}=\underline{T}$. If $\underline{T}^{\prime}$ denotes the class of torsion free abelian groups then $L T^{\prime}=\underline{T}$. 
We will now charactcrize the class $V\left(T^{\prime}\right)$. We will show that

$$
V\left(\underline{T}^{\prime}\right)=\left\{M \epsilon \underline{T}^{\prime} \mid J(M)=0\right\} .
$$

Let $M \in V\left(\underline{T}^{\prime}\right)$. We will show that 0 is the only small submodule of $M$. Then it follows that $J(M)=0$. Suppose on the contrary $0 \neq N \ll M$. Since $V\left(\underline{T}^{\prime}\right) \subseteq \underline{T}^{\prime}$ we have $M \epsilon \underline{T}^{\prime}$. Hence $N \epsilon \underline{T^{\prime}}$. This means there is a copy of $\mathbf{Z}$ in $N$. Consider the subgroup $2 \mathbf{Z}$ of $\mathbf{Z}$. From $2 \mathbf{Z} \leq \mathbf{Z} \leq N \ll M$ we get $2 Z \ll M$. Now, $M / 2 Z$ has non-zero 2 torsion, contradicting the fact that $M \epsilon V\left(\underline{T}^{\prime}\right)$. Conversely any $M \epsilon \underline{T}^{\prime}$ with $J(M)=0$ is cicarly in $V\left(T^{\prime}\right)$ because then 0 is the only small submodule of $M$ and $M / 0 \simeq$ $M \epsilon \underline{T}^{\prime}$. This proves (5). From (5) we see that the inclusion $V\left(\underline{T}^{\prime}\right) \subseteq \underline{T}^{\prime}$ is a strict inclusion, because $Q \epsilon T^{\prime}$ but $Q \notin V\left(T^{\prime}\right)$ since $J(Q)=Q$. We included information on the classes $L \underline{T}, V \underline{T}, L \underline{T}^{\prime}$ and $V \underline{T}^{\prime}$ to complete the examples discussed in 1.7 .

\section{References}

1. A. W. ChatTERS, A characterization of right Noetherian rings, Quarterly J. Math. Oxford 33 (1982), 65-69.

2. Dinh Van Huynh and Phan Dan, On rings with restricted minimum condition, Arch. Math. 51 (1988), 313-326.

3. Diniz Van Huyna, Nguyen V. Dung and Patrick F. Smirh, Rings characterized by their right ideals or cyclic modules, Proceedings of the Edinburgh Mathematical Society 32 (1989), 355-362.

4. Patrick F. Smith, Din Van Huynh and Nguyen V. Dunc, A characterization of Noctherian modules, Quart. J. Math. Oxford 41 (1990), 225-235.

5. Din Van Huynh, Nguyen V. Dung, and Patrick F. Smith, A characterization of rings with Krull dimension, J. Alg. 132 (1990), 104-112.

6. Ding Van Huynh and NGUYen V. Dung, A characterization of artinian rings, Glasgow Math. J. 30 (1988), 67-73.

7. B. Sarath and $K$. Varadarajan, Dual Goldie dimension II, Communications in Alg. 7 (1979), 1885-1899.

8. P. F. SMITH, Some rings which are characterized by finitely generated modules; Quart. J. Math. Oxford 29 (1978), 101-109.

9. P. F. SMITH, Rings characterized by their cyclic modules, Canadian J. Math. 30 (1978), 98-111.

10. P. VAMOS, The dual of the notion of finitely generated, J. London Math. Soc. 43 (1969). 
11. K. Varadarain, Dual Goldie dimension, Communications in Alg. $7(1979), 565-610$.

12. K. Varadarajan, Modules with supplements, Pac. J. of Math. 82 (1979), 559-564.

13. K. Varadarajan and P. R. Wani, Modules over endomorphism rings I!, Acti Math. Hangarica 53 (1989), 309-337.

14. K. Varadarajan, Hopfian and co-Hopfian objects (to appear).

Department of Mathematics and Statistics

The University of Calgary

2500 University Drive N.W.

Calgary, Alberta

CANADA T2N $1 \mathrm{~N} 4$

Primera versió rebuda el 15 de Novembre de 1991, darrera versió rebuda el 2 de Març de 1992 